\title{
Obligatorische Meldepflicht für Masern in der Schweiz: starker Anstieg der Anzahl Fälle im Frühjahr 2003
}

\author{
J.-L. Richard ${ }^{a}$, K. Boubaker ${ }^{b}$, M. Doutaz ${ }^{c}$, G. Schubiger
}

\section{Zusammenfassung}

Seit Anfang Jahr ist in der Schweiz eine starke Masernepidemie zu beobachten. Die Anzahl der im Rahmen der obligatorischen Meldepflicht gemeldeten Fälle belief sich von Januar bis Anfang Juni auf 464. Die effektive Zahl der Fälle liegt jedoch höher, da nicht alle Kranken den Arzt aufsuchen und nicht alle Patientinnen und Patienten gemeldet werden. Die am meisten betroffenen Kantone sind Schwyz, Wallis, Freiburg, Zürich und Genf. 79\% der Patientinnen und Patienten waren jünger als 16 Jahre, wovon mehr als 90\% nicht geimpft waren. Es wurden 51 Komplikationen und 26 Hospitalisierungen gemeldet. Als Beispiele werden zwei Fallbeschreibungen aufgeführt. Aufgrund einer noch ungenügenden Durchimpfung der Bevölkerung kann das Virus in der Schweiz weiterhin zirkulieren und periodisch Epidemien verursachen.

\section{Einleitung}

Die Masern, eine stark ansteckende Krankheit, machten vor Einführung der Impfung im Jahr 1976 die meisten Kinder in der Schweiz durch. Die manchmal fälschlicherweise als harmlos bezeichnete Krankheit führt zu einem allgemeinen Exanthem mit starkem Fieber, oft begleitet von Husten, Rhinitis, Konjunktivitis und KoplikFlecken, und kann gravierende, selten auch tödliche Folgen haben. Mit der Verbreitung der Masernimpfung und später der MMR-Impfung (Masern, Mumps, Röteln) ging die Anzahl Fälle in der Schweiz stark zurück. Damit vollzog sich ein Wechsel von einer epidemischen Übertragung mit der ständigen Zirkulation des Virus und epidemischen Spitzen alle 2 bis 4 Jahre [1] zu einer sporadischen Übertragung mit isolierten Fällen und seltenen lokalen Ausbrüchen. Die un- genügende Durchimpfung gegen Masern kann aber nach wie vor periodisch zur Entwicklung von stärkeren Epidemien führen, wie dies seit Anfang Jahr der Fall ist.

Der vorliegende Artikel beschreibt anhand der Daten der obligatorischen Meldungen die Masernepidemie des Frühjahrs 2003. Dabei wird vor allem auf deren Ursachen und Folgen in bezug auf Komplikationen eingegangen, namentlich mit zwei kurzen Fallbeschreibungen (eine masernbedingte Enzephalitis und eine Masernerkrankung mit Lungenentzündung).

\section{Material und Methoden}

Die individuelle Meldung von klinischen Masernfällen ist für die Ärzteschaft seit 1999 obligatorisch. Alle Fälle mit Fieber, makulopapulösem Exanthem und Husten, Rhinitis oder Konjunktivitis müssen dem Kantonsarzt gemeldet werden. Ebenfalls 1999 ging man von der Kollektivmeldung zur individuellen Meldung aller in Labors bestätigten Masernfälle über. Die Formulare zur Erst- und Ergänzungsmeldung für Ärzteschaft und Labors können beim Kantonsarzt bezogen oder auf der Website des Bundesamts für Gesundheit (http://www.bag.admin.ch/infreporting/ forms/d/index.htm) heruntergeladen werden. Wir haben die Fälle analysiert, die dem BAG von Januar bis Anfang Juni 2003 gemeldet wurden (Wochen 1 bis 22).

Das Sentinella-Meldesystem überwacht die Masern seit 1986 ebenfalls. Auf freiwilliger Basis melden rund 250 Grundversorger dem BAG wöchentlich die klinischen Fälle bestimmter Krankheiten, darunter auch die Masern. Aufgrund der unterschiedlichen Sensitivität und Ausformung der beiden Systeme haben wir die beiden Datensammlungen nicht zusammen analysiert. 
Abbildung 1

Anzahl der 2003 gemeldeten Masernfälle in der Schweiz.

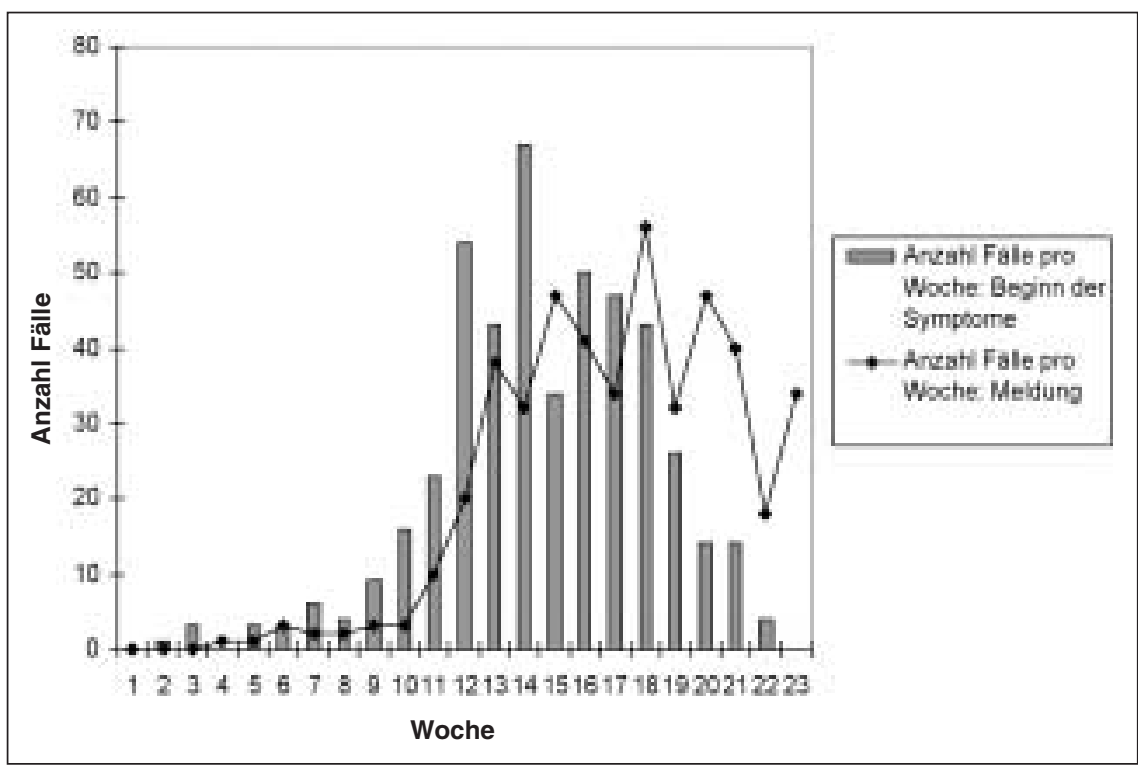

Abbildung 2

Verteilung der 2003 gemeldeten Masernfälle in der Schweiz (Wochen 1 bis 22).

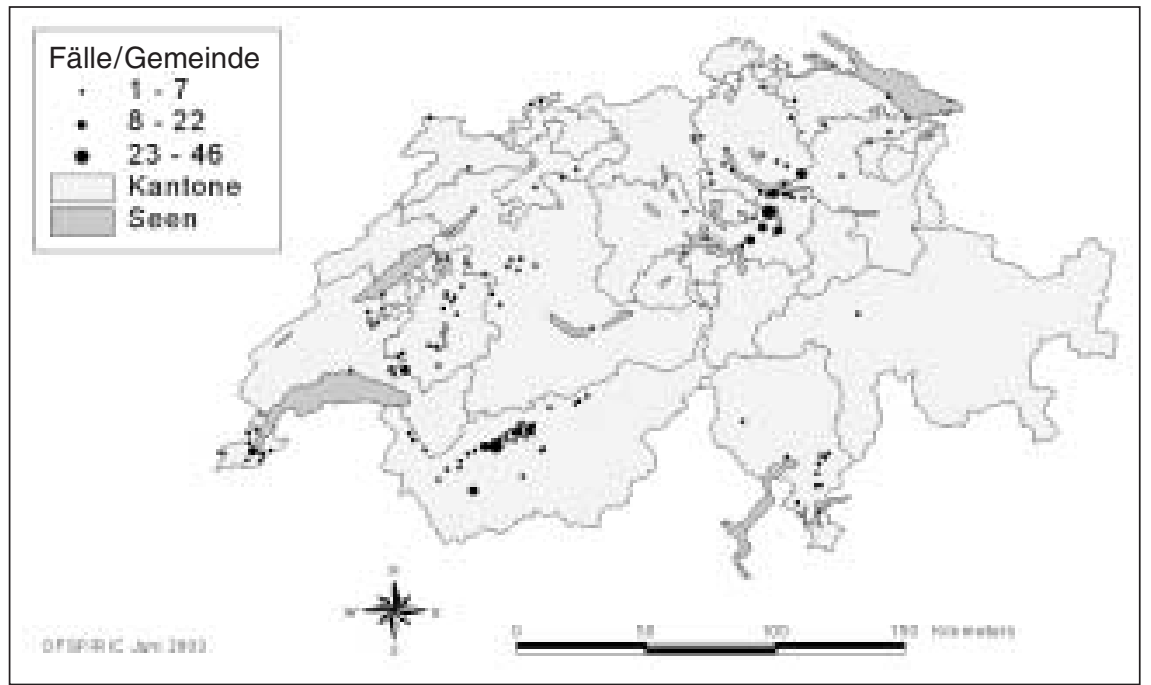

\section{Ergebnisse}

\section{Anzahl gemeldeter Fälle}

In den ersten fünf Monaten des Jahres 2003 wurden 464 Masernfälle gemeldet. Bei 436 Fällen $(94,0 \%)$ ging auch eine Ergänzungsmeldung ein. Die epidemische Kurve zeigt einen starken Anstieg ab der Woche 7 mit einer Spitze von 67 Fällen in Woche 14 (Abb. 1). Das Maximum der Epidemie scheint gegenwärtig überschritten zu sein.
Die gegenwärtig herrschende Masernepidemie ist durch eine Vielzahl lokaler oder regionaler Ausbrüche gekennzeichnet und damit durch eine grosse räumliche Heterogenität in bezug auf die ganze Schweiz (Abb. 2). Allein fünf Kantone verzeichneten $85 \%$ der Fälle: Wallis (162 Fälle, $35 \%$ ), Schwyz (98 Fälle, 21\%), Freiburg (53 Fälle, 11\%), Zürich (42 Fälle, 9\%) und Genf (39 Fälle, $8 \%)$. Insgesamt wurden in 18 Kantonen Fälle gemeldet. Wie in Abbildung 2 ersichtlich, gibt es auch innerhalb der am meisten betroffenen Kantone lokale Häufungen.

Es wurden fast gleichviel weibliche (221/456, 47,6\%) wie männliche Patienten gemeldet. Das mittlere Alter der Fälle lag bei 10,3 Jahren. 30\% der Fälle waren 5-9 Jahre alt, 26\% 10-14 Jahre, 17\% 15-19 Jahre, 17\% 1-4 Jahre, 8\% 20 Jahre und älter und $2 \%$ jünger als $1 \mathrm{Jahr}$ (Abb. 3). Die Altersverteilung war für die drei Hauptherde unterschiedlich. Im Kanton Schwyz erkrankten vor allem Kinder von 5 bis 9 Jahren an Masern (46\% der Fälle im Kanton). Im Wallis waren vor allem grössere Kinder und Jugendliche betroffen (70\% der Fälle bei 10- bis 19jährigen), während im Kanton Freiburg vor allem Kinder im Alter von 5 bis 14 Jahren erkrankten (75\% der Fälle). Diese Unterschiede sind zum Teil auf die Art des Erstherdes bei diesen Ausbrüchen zurückzuführen: eine Primarschule in Freiburg und ein Gymnasium im Wallis.

Von den 395 Fällen (85\%) mit bekanntem Impfstatus waren 38 (9,6\%) gegen Masern geimpft, davon 34 (91\%) mit einer einzigen Dosis, drei mit zwei Dosen und ein Fall mit einer unbekannten Anzahl Dosen. Zehn der mit einer Dosis geimpften Fälle sind vom Labor bestätigt, desgleichen ein Fall mit zwei Impfdosen. Sieht man von den unter einjährigen Kindern und den über 20jährigen Personen ab, ist der Anteil der geimpften Fälle bezogen auf das Alter relativ stabil (Abb. 3).

Bei 136 (29\%) der klinischen Masernfälle wurde eine Laboruntersuchung veranlasst, mit der 125 Fälle, d.h. $27 \%$ der insgesamt gemeldeten Fälle, bestätigt werden konnten. Die Bestimmung des Genotyps des Virus gelang in 16 Fällen: sieben Fälle in Schwyz, sieben im Wallis und zwei in Genf. Die in Schwyz und Genf zirkulierenden Viren gehören zum Genotyp D8, diejenigen im Wallis zum Genotyp D5. Der erstere ist in Indien, Nepal und Äthiopien endemisch, letzterer in Japan und Thailand [2]. Die Labordaten bestätigen den asiatischen Ursprung der gemeldeten Fälle im Kanton Schwyz. Der Indexfall ist ein Kind aus diesem Kanton, das kurz nach der Rückkehr aus den in Indien verbrachten Weihnachtsferien erkrankte. Das Kind übertrug die 
Abbildung 3

Altersverteilung und Impfstatus der dem Bundesamt für Gesundheit 2003 gemeldeten Masernfälle (Wochen 1 bis 22).

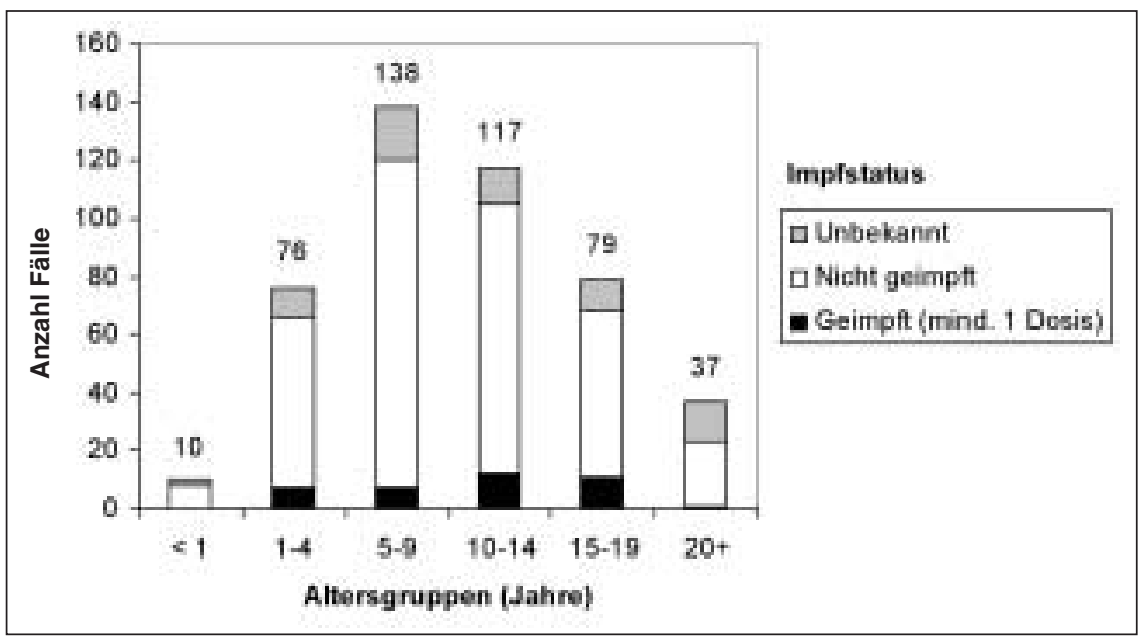

Krankheit auf die Schülerinnen und Schüler der Privatschule, die es besucht. Diese fuhren kurz darauf für eine Skiwoche in eine andere Region des Kantons Schwyz, was zur Verbreitung der Krankheit im Kanton beitrug. Zwischen den Fällen aus Schwyz und jenen aus Genf konnte keine epidemiologische Verbindung hergestellt werden.

\section{Komplikationen}

Während der 22 ersten Wochen des Jahres 2003 wurde bei 51 (11,0\%) der 464 gemeldeten Fälle eine Komplikation beobachtet. Es handelte sich um 3 Fälle von Enzephalitis, 14 Pneumonien, 3 Fälle mit Verdacht auf Pneumonie, 11 Fälle von Otitis, 1 Delirium, 1 Myokarditis, 1 Fall mit generalisierten tonischen Konvulsionen, 1 Gingivostomatitis, 1 Hepatitis mit Verdacht auf Appendizitis, 1 Otitis mit Hepatitis und 14 Hospitalisierungen ohne Nennung von Komplikationen. 26 (5,6\%) Personen wurden hospitalisiert, davon eine Person für nahezu 6 Wochen wegen einer Enzephalitis. Kein Todesfall war zu verzeichnen.

Das Komplikationsrisiko nimmt mit zunehmendem Alter zu. Es steigt von 6,6\% für Kinder im Alter von 1 bis 4 Jahren auf 24,3\% für Erwachsene von 20 und mehr Jahren. 32 Kinder unter 16 Jahren $(8,9 \%)$ hatten eine Komplikation im Vergleich zu 18 Erwachsenen (18,8\%) $(\mathrm{p}=0,006)$. Die Patienten mit Enzephalitis waren 10, 10 und 16 Jahre alt. Zwei geimpfte Fälle $(5,3 \%)$ hatten eine Komplikation im Vergleich zu 43 nicht geimpften Patienten (12\%) und $6 \mathrm{~Pa}$ tienten $(8,7 \%)$ mit einem unbekannten Impfstatus (statistisch kein signifikanter Unterschied).
Fallbeispiel 1: Masernenzephalitis bei einem Jugendlichen

Ein nicht geimpfter 17jähriger Schüler mit vorgängig guter Gesundheit und unauffälliger Vorgeschichte musste am 25. März 2003 wegen Bewusstseinsverlust mit tonisch-klonischen Krampfanfällen notfallmässig ins Spital eingewiesen werden.

Nach Angaben der Familie hatte der Patient seit 7 Tagen Fieber, Kopfschmerzen, Myalgien, eine beidseitige Konjunktivitis, Rhinitis sowie seit 4 Tagen ein Exanthem am ganzen Körper. Das Exanthem trat zuerst am Kopf auf und breitete sich in der Folge rasch auf den Stamm und die Extremitäten aus. Nach primär gutartigem Krankheitsverlauf kam es zu einem Bewusstseinsverlust mit tonisch-klonischen Krampfanfällen. Bekannt ist ein Kontakt zu einem wahrscheinlichen Masernfall ungefähr 10 Tage vor Beginn der Symptome.

Bei Spitaleintritt war der Patient in schlechtem Allgemeinzustand, bewusstlos, afebril $\left(37^{\circ} \mathrm{C}\right)$ und wies eine Hypertonie $(170 / 70 \mathrm{mmHg}$ ) und Sinusbradycardie von 45/Min. auf. Vor allem im Gesicht und am Stamm bestand ein makulopapulöses Exanthem mit Petechien und Schwellung der Augenlider. Die neurologische Untersuchung zeigte einen komatösen Patienten mit kortiko-bulbären und kortiko-spinalen Zeichen (Hyperreflexie, Spastizität, bilateraler Babinsky). Die übrige klinische Untersuchung erbrachte keine Besonderheiten. Die Laboruntersuchungen ergaben unter anderem positive Masern-IgM und -IgG und eine leichte Thrombozytopenie. Im MRI vom 26. März 2003 zeigte sich eine Pansinusitis, in jenem vom 31. März 2003 eine leichte diffuse Hyperdensität parieto-okzipital beidseits der weissen Hirnsubstanz, vereinbar mit einer Enzephalitis.

Aufgrund der klinischen Befunde und der ergänzenden Untersuchungen wurde die Diagnose einer Masernenzephalitis gestellt. Unter symptomatischer Behandlung und respiratorischer Unterstützung verbesserte sich der Zustand langsam. Das Bewusstsein klärte zunehmend auf und der Patient konnte am 28. März 2003 extubiert werden. $\mathrm{Zu}$ diesem Zeitpunkt ergab die neurologische Untersuchung einen Glasgow-ComaScore von 15, eine Dysmetrie und eine persistierende leichte Desorientierung. Der weitere Verlauf war günstig. Bei Spitalentlassung persistierten noch eine leichte psychomotorische Verlangsamung, kortikobulbäre Zeichen mit Palmomentalreflex und Störungen der Augenmotilität sowie eine intermittierende BradyDysrhythmie fronto-temporal rechts bei normaler Grundaktivität im EEG. Ein nach dem 
Abbildung 4

Intubierter Patient mit ZVK rechts und Magensonde. Ausgedehnte grobfleckige, teilweise konfluierende pulmonale Infiltrate beidseits betont im linken Oberlappen zentral, im rechten Oberlappen paramediastinal sowie in beiden Unterlappen medial. Kleiner Pleuaraerguss rechts mit Obliteration des Sinus phreniko-kostalis lateral. Kardial kompensiert bei normaler Herzgrösse.

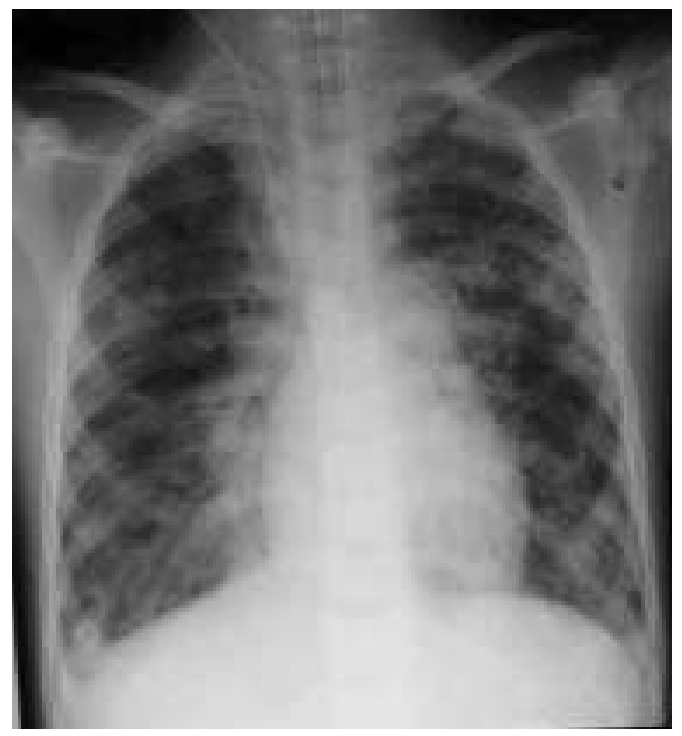

Austritt erfolgter neuropsychologischer Status zeigt eine inzwischen vollständige Normalisierung auf.

\section{Fallbeispiel 2: Schwere pulmonale Komplikation nach Masern}

Ein 10jähriger, MMR-ungeimpfter Knabe wurde 14 Tage nach Beginn einer klassischen Masernerkrankung und nach Abblassen des Exanthems wegen Tachy-Dyspnoe und Husten in hochfebrilem Zustand zugewiesen. Zur Behandlung wurde Vitamin A verabreicht und wegen deutlicher laborchemischer Hinweise auf eine bakterielle Superinfektion eine antibiotische Behandlung begonnen. Am folgenden Tag musste der Patient bei drohender respiratorischer Dekompensation intubiert und künstlich beatmet werden. Radiologisch zeigten sich zunehmend pulmonale diffus-fleckige interstitielle Infiltrate (vgl. Abb. 4) Ein bakterieller Erregernachweis ist nicht gelungen. Nach 9 Tagen Intensivtherapie mit Sauerstoffbedarf bis 100\% und Vasoaktivaunterstützung konnte problemlos extubiert werden, und der Knabe wurde nach insgesamt 23tägiger Hospitalisation entlassen. Aufgrund des klinischen Verlaufes wird die Krankheit als vital bedrohliche Masernpneumonie mit möglicher bakterieller Superinfektion beurteilt.

\section{Diskussion}

In den Wochen 1 bis 22 des Jahres 2003 wurde in der Schweiz ein starker Anstieg der dem BAG gemeldeten Masernfälle verzeichnet. Die Anzahl der bestätigten Fälle, bei denen die Symptome in den ersten fünf Monaten des Jahres begannen, lag für 2000, 2001 und 2002 bei nur 9 bzw. 27 und 27 im Vergleich mit 464 Fällen für das Jahr 2003. Diese Zahl stellt dabei nur einen Bruchteil der gesamten Anzahl Masernfälle seit Jahresbeginn in der Schweiz dar: Zum einen, weil nicht alle Erkrankten zum Arzt gehen, vor allem bei starken Ausbrüchen, zum andern, weil nicht alle beim Arzt behandelten Fälle gemeldet werden dies in erster Linie deshalb, weil wahrscheinlich ein Teil der Ärzteschaft nicht weiss, dass Masern seit 1999 der obligatorischen Meldepflicht unterliegen. In Extremfällen kann die Kombination dieser beiden Faktoren zu einer beträchtlichen Unterschätzung der wirklichen Situation führen. So wurden etwa von den 42 in einer Schwyzer Schule aufgetretenen Fällen nur deren zwei mit Meldeformularen dem Kantonsarzt und dem BAG gemeldet. Gemäss einer italienischen Studie liefert die obligatorische Meldung von Masernfällen bei Kindern beinahe viermal weniger Fälle, als aufgrund der Extrapolationen der Meldungen durch die Sentinella-Kinderärzte zu erwarten wären [3]. Für die Masernepidemie von 1999 bis 2000 in den Niederlanden schätzt man die Anzahl der tatsächlichen Erkrankungen auf rund zehnmal höher als die 3292 berichteten Fälle [4].

Die Einführung einer Kleinkinderimpfung kann bei ungenügender Durchimpfung das mittlere Alter der Erkrankten erhöhen. Mit 21\% der Fälle mit Alter 16 und mehr und einem mittleren Alter von 10,3 Jahren liegt das Alter der jetzigen Epidemie relativ hoch im Vergleich zu einer Situation ohne Impfungen. Die im Rahmen der obligatorischen Meldepflicht erhaltenen Daten sind jedoch noch zu wenig umfassend (1999-2003), als dass eine mögliche Altersverschiebung bei Masernfällen beobachtet werden könnte. Die seit 1986 verfügbaren SentinellaDaten zeigen keine Tendenz in diese Richtung.

In den meisten Fällen erholen sich Masernpatientinnen und -patienten ohne Komplikationen rund eine Woche nach Auftreten des Exanthems [5]. Im Durchschnitt tritt auf zehn Fälle eine Komplikation auf, die in Industrieländern in ungefähr einem von 2000 Fällen zum Tode führt [6]. Die am meisten gefürchteten Komplikationen sind Enzephalitis und Pneumonie. Er- 
stere treten in rund einem von 500 bis 5000 Fällen auf, letztere in einem Fall von 17 bis 100 [6]. Die in der gegenwärtigen Epidemie beobachtete Komplikationsrate entspricht den Werten aus der Literatur. Solche Vergleiche sind jedoch heikel, da die Daten aus der passiven Überwachung möglicherweise durch eine unterschiedliche Meldehäufigkeit von leichteren Fällen und solchen mit Komplikationen verfälscht werden können. Die Häufigkeit von Enzephalitis ist mit einem Fall auf 155 gemeldete Masernerkrankungen jedoch stark erhöht. Eine vierte Enzephalitis wurde zudem von einem Sentinella-Arzt gemeldet. Die Frage bleibt offen, ob dies als Zufall zu werten ist, als Indiz für eine massive Unterdeklarierung von Fällen ohne Komplikationen oder als Hinweis auf eine besonders starke Virulenz der aktuellen Masernviren. Trotz der verschiedenen Virustypen lassen sich keinerlei Anzeichen für eine verminderte Wirkung der Masernimpfstoffe feststellen. Die Impfung schützt nicht nur zuverlässig gegen Masern; unsere Daten scheinen auch nahezulegen, dass sie im Falle einer Erkrankung zu einem gewissen Grad Komplikationen verhindern kann. Die Daten aus der passiven Überwachung sind allerdings nicht ideal für das Bestimmen von Komplikationsraten, und unsere Ergebnisse sind statistisch nicht signifikant. In Übereinstimmung mit der Literatur konnten wir eine altersabhängige $\mathrm{Zu}$ nahme der Komplikationsrate zeigen [7].

Das BAG empfahl seit 1976 die Masernimpfung im Alter von 12 Monaten. Die Kombinationsimpfung MMR wurde seit 1985 für Kinder im Alter von 15-24 Monaten empfohlen mit einer Nachholimpfung im Alter von 12 bis 15 Jahren. 1996 wurde eine Zweitdosis für Kinder von 4 bis 7 Jahren eingeführt [8], und 2001 senkte man das Alter der Verabreichung der Erstdosis von 15 auf 12 Monate, jenes für die Zweitdosis auf 15 bis 24 Monate [9]. Nachholimpfungen sind bei Schuleintritt und -austritt vorgesehen sowie zu einem beliebigen Zeitpunkt bei Erwachsenen. Trotz dieser Empfehlungen und Kampagnen zur Förde- rung von Routineimpfungen in den Jahren 1987 und 2002 blieb die Durchimpfungsrate für Masern in der Schweiz im vergangenen Jahrzehnt für Kleinkinder stabil im Bereich von 80\% [10, 11]. In den Jahren 2000 bis 2002 betrug die Durchimpfung mit einer Dosis für Kinder zwischen 24 und 35 Monaten $81 \%$, für 5- bis 7jährigen Kinder $89 \%$ und für Jugendliche $94 \%$ [12]. Um die Zirkulation des Virus zu unterbrechen und die Masern gemäss den Zielen der WHO bis im Jahr $2010 \mathrm{zu}$ eliminieren [13], müssen mindestens 95\% der Kinder zweimal geimpft sein.

Die aus dem Wallis gemeldeten Fälle lassen vermuten, dass die fehlenden Impfungen grösstenteils auf die Entscheidung einer Minderheit von Eltern zurückgehen, Ärzte aufzusuchen, die die MMR-Impfung nicht empfehlen oder dieser gar ablehnend gegenüberstehen. Die gemeldeten Fälle konzentrieren sich in der Tat tendenziell in der Umgebung einiger alternativer Ärzte. Der Ausbruchsherd in Schwyz befindet sich in einer Privatschule mit einer alternativen Pädagogik. 42 der 46 Kinder zogen sich hier innerhalb von einigen Wochen die Masern zu.

Die ungenügende Durchimpfung vor allem bei Kleinkindern und das Vorkommen von nicht immunen Bevölkerungsnischen führen in der Schweiz periodisch zum Auftreten von Masernepidemien. Mit der Schaffung des SentinellaSystems 1986 konnten in der Schweiz bereits 1987 und 1997 grössere Masernepidemien nachgewiesen werden; die geschätzte Anzahl Fälle betrug 10500 bzw. 6400 [14]. Eine verstärkte Förderung der MMR-Impfung ist nötig, um die Masern in der Schweiz zu eliminieren, wie dies in Finnland seit 1996 der Fall ist [15].

\section{Dank}

Die Autoren danken Pierre-Alain Raeber, Hanspeter Zimmermann, Catherine Bourquin, Daniel Koch und Hans-Peter Roost vom BAG für ihre kritischen Kommentare. 


\section{Literatur}

1 Gabutti G, Rota MC, Salmaso S, Bruzzone BM, Bella A, Crovari P and the Serological Study Group. Epidemiology of measles, mumps and rubella in Italy. Epidemiol Infect 2002;129: 543-50.

2 Rota PA, Bellini WJ. Update on the global distribution of genotypes of wild type measles viruses. J Infect Dis 2003;187(Suppl 1):S270-6.

3 Ciofi degli Atti ML, Salmaso S, Bella A, Arigliani R, Gangemi M, Chiamenti G, et al. Pediatric sentinel surveillance of vaccine-preventable diseases in Italy. Pediatr Infect Dis J 2002;21:763-8.

4 Van den Hof S, Conyn-van Spaendonck MAE, van Steenbergen JE. Measles epidemic in the Netherlands, 1999-2000. J Infect Dis 2002;186: 1483-6.

5 Gershon AA. Measles virus (rubeola). In: Mandell GL, Bennett JE, Dolin R (eds.). Principles and practice of infectious diseases. $5^{\text {th }}$ edition. Philadelphia: Churchill Livingstone; 2000. p. 1801-9.

6 Bundesamt für Gesundheit und Schweizerische Kommission für Impffragen. Prävention von Masern, Mumps und Röteln. In: Bundesamt für Gesundheit (Hrsg.). Infektionskranheiten: Diagnose und Bekämpfung. Bern: Bundesamt für Gesundheit; 2003. Supplementum XII (im Druck).

7 Miller DL. Frequency of complications of measles. Br Med J 1963;11:75-8.

8 Bundesamt für Gesundheit und Schweizerische Kommission für Impffragen. Impfungen für routinemässige Schutzimpfungen. In: Bundesamt für Gesundheit (Hrsg.). Infektionskranheiten: Diagnose und Bekämpfung. Bern: Bundesamt für Gesundheit; 1996. Supplementum VIII.
9 Bundesamt für Gesundheit und Schweizerische Kommission für Impffragen. Impfungen für routinemässige Schutzimpfungen. In: Bundesamt für Gesundheit (Hrsg.). Infektionskranheiten: Diagnose und Bekämpfung. Bern: Bundesamt für Gesundheit; 2001. Supplementum VIII.

10 Minder C, Steffen R. Repräsentative Erhebung zur Durchimpfung in der Schweiz 1991. Bull BAG/OFSP 1992;32:504-7.

11 Bundesamt für Gesundheit. Repräsentative Erhebung zur Durchimpfung in der Schweiz 1998. Bull BAG/OFSP 1999;20:356-61.

12 Persönliche Mitteilung Phung Lang, Zürich.

13 Spika JS, Wassilak S, Pebody R, Lipskaya G, Deshevoi S, Güris D, et al. Measles and rubella in the World Health Organization European Region: diversity creates challenges. J Infect Dis 2003:187(Suppl 1):S191-7.

14 Paget WJ, Zimmermann H, Vorkauf H, Groupe de travail Sentinella. Epidémie de rougeole en Suisse en 1997: conséquences sur l'élimination de la rougeole à l'horizon 2007. Eurosurveillance 2000;5:17-20.

15 Bundesamt für Gesundheit. Elimination der Masern in Finnland. Bull BAG/OFSP 2003;16:278. 